\title{
Correction to: Embedding theorems for Sobolev and Hardy-Sobolev spaces and estimates of Fourier transforms
}

\section{I. Kolyada ${ }^{1}$}

Accepted: 16 September 2021 / Published online: 25 September 2021

(c) Fondazione Annali di Matematica Pura ed Applicata and Springer-Verlag GmbH Germany, part of Springer Nature 2021

\section{Correction to: Annali di Matematica Pura ed Applicata (1923 -) (2019) 198:615-637 DOI: https://doi.org/10.1007/s10231-018-0792-2}

The original version of this article was published on 25th December 2018 in Volume 198, issue 2, page 615-637, contained errors in the formulation of Theorem 6.3 and in the proof of Theorem 6.5 in paper [4].

There were some errors in the formulation of Theorem 6.3 and in the proof of Theorem 6.5 in our paper [4]. The part of [4] beginning from Theorem 6.3 (inclusive) till the end of Section 6 should be replaced by the following corrected text.

Theorem 0.1 Let $f \in W_{1}^{1}\left(\mathbb{R}^{n}\right)(n \geq 3)$. For a fixed $\xi_{j} \in \mathbb{R}$, let $\left(\widehat{f}_{\xi_{j}}\right)^{*}(s)$ be the nonincreasing rearrangement of the function

$$
\widehat{\xi}_{j} \mapsto\left|\widehat{f}\left(\xi_{j}, \hat{\xi}_{j}\right)\right|, \hat{\xi}_{j} \in \mathbb{R}^{n-1}
$$

Set

$$
\Phi_{j}(s, t)=\sup _{\left|\xi_{j}\right| \geq t}\left(\widehat{f}_{\xi_{j}}\right)^{*}(s) \quad(s, t>0) .
$$

Then,

$$
\sum_{j=1}^{n} \int_{0}^{\infty} \int_{0}^{\infty}(s t)^{1 / n-1} \Phi_{j}(s, t) d s d t \leq c\|\nabla f\|_{1} .
$$

Proof We estimate the first term of the sum in (0.1). Set $\varphi_{h}(x)=\Delta_{1}(h) f(x)$. Then,

The original article can be found online at https://doi.org/10.1007/s10231-018-0792-2.

V. I. Kolyada

viktor.kolyada@gmail.com

1 Department of Mathematics, Karlstad University, Universitetsgatan 1, 65188 Karlstad, Sweden 


$$
\widehat{\varphi_{h}}(\xi)=\widehat{f}(\xi)\left(e^{2 \pi i h \xi_{1}}-1\right) .
$$

Let $t>0$ and $\tau=1 / t$. Assume that $\left|\xi_{1}\right| \geq t$. Then,

$$
\begin{aligned}
\frac{1}{\tau} \int_{0}^{\tau}\left|e^{2 \pi i h \xi_{1}}-1\right| d h & \geq \frac{1}{\tau} \int_{0}^{\tau}\left(1-\cos \left(2 \pi \xi_{1} h\right)\right) d h \\
& =1-\frac{\sin \left(2 \pi \xi_{1} \tau\right)}{2 \pi \xi_{1} \tau} \geq 1-\frac{1}{2 \pi\left|\xi_{1}\right| \tau} \geq 1-\frac{1}{2 \pi} .
\end{aligned}
$$

It follows that

$$
\frac{2}{\tau} \int_{0}^{\tau}\left|\widehat{\varphi_{h}}(\xi)\right| d h \geq|\widehat{f}(\xi)| \quad \text { if } \quad\left|\xi_{1}\right| \geq t .
$$

By $(0.2)$ and $[4,(2.2)]$, for a fixed $\xi_{1}$ with $\left|\xi_{1}\right| \geq t$ and any $s>0$

$$
\left(\widehat{f}_{\xi_{1}}\right)^{*}(s) \leq \frac{2}{\tau s} \int_{0}^{\tau} \sup _{\text {mes }_{n-1} E=s} \int_{E}\left|\widehat{\varphi_{h}}(\xi)\right| d \widehat{\xi}_{1} d h .
$$

Let $1<q<2$. By Hölder's inequality, for any set $E \subset \mathbb{R}^{n-1}$ with mes ${ }_{n-1} E=s$ and any fixed $\xi_{1} \in \mathbb{R}$

$$
\frac{1}{s} \int_{E}\left|\widehat{\varphi_{h}}(\xi)\right| d \widehat{\xi}_{1} \leq s^{-1 / q^{\prime}}\left(\int_{\mathbb{R}^{n-1}}\left|\widehat{\varphi}_{h}(\xi)\right|^{q^{\prime}} d \widehat{\xi}_{1}\right)^{1 / q^{\prime}} .
$$

Fix $h>0$ and $\xi_{1} \in \mathbb{R}$ and consider the function

$$
\psi_{h, \xi_{1}}\left(\hat{x}_{1}\right)=\int_{\mathbb{R}} \varphi_{h}(x) e^{-2 \pi i \xi_{1} x_{1}} d x_{1}, \quad \hat{x}_{1} \in \mathbb{R}^{n-1} .
$$

Applying the Hausdorff-Young inequality to the function $\psi_{h, \xi_{1}}$, we obtain

$$
\begin{aligned}
& \left(\int_{\mathbb{R}^{n-1}}\left|\widehat{\psi \psi_{h, \xi_{1}}}\left(\widehat{\xi}_{1}\right)\right|^{q^{\prime}} d \widehat{\xi}_{1}\right)^{1 / q^{\prime}} \\
& \quad \leq\left(\int_{\mathbb{R}^{n-1}}\left(\int_{\mathbb{R}}\left|\varphi_{h}(x)\right| d x_{1}\right)^{q} d \widehat{x}_{1}\right)^{1 / q} .
\end{aligned}
$$

It is clear that $\widehat{\varphi_{h}}(\xi)=\widehat{\psi_{h, \xi_{1}}}\left(\widehat{\xi}_{1}\right)$ for any $\widehat{\xi}_{1} \in \mathbb{R}^{n-1}$ (where $h$ and $\xi_{1}$ are fixed). Thus, using (0.3), (0.4), and (0.5), we get that for any $s, t>0$, any $\xi_{1}$ satisfying $\left|\xi_{1}\right| \geq t$, and any $1<q<2$

$$
\left(\widehat{f}_{\xi_{1}}\right)^{*}(s) \leq 2 t s^{-1 / q^{\prime}} \int_{0}^{1 / t} \mu_{q}(h) d h,
$$

where $\mu_{q}(h)=\left\|\Delta_{1}(h) f\right\|_{L^{q}\left[L^{1}\right]}$. It follows that

$$
\Phi_{1}(s, t) \leq 2 t s^{-1 / q^{\prime}} \int_{0}^{1 / t} \mu_{q}(h) d h \quad \text { for any } 1<q<2, s, t>0 .
$$

Observe also that 


$$
\mu_{q}(h)=\left\|\Delta_{1}(h) f\right\|_{L^{q}\left[L^{1}\right]} \leq c\left\|\Delta_{1}(h) f\right\|_{L^{q, 1}\left[L^{1}\right]} .
$$

Set

$$
I^{\prime}=\int_{0}^{\infty} s^{1 / n-1} \int_{0}^{s^{1 /(n-1)}} t^{1 / n-1} \Phi_{1}(s, t) d t d s .
$$

Taking into account that $n \geq 3$, choose $n^{\prime}<q<(n-1)^{\prime}$. Then, $1<q<2$ and $1 / n<1 / q^{\prime}<1 /(n-1)$. Thus, applying (0.6), [4, Theorem 4.2], and using (0.7), we obtain

$$
\begin{aligned}
I^{\prime} & \leq 2 \int_{0}^{\infty} s^{1 / n-1 / q^{\prime}-1} \int_{0}^{s^{1 /(n-1)}} t^{1 / n} \int_{0}^{1 / t} \mu_{q}(h) d h d t d s \\
& \leq 2 \int_{0}^{\infty} t^{1 / n} \int_{0}^{1 / t} \mu_{q}(h) d h \int_{t^{n-1}}^{\infty} s^{1 / n-1 / q^{\prime}-1} d s d t \\
& =c \int_{0}^{\infty} t^{1-(n-1) / q^{\prime}} \int_{0}^{1 / t} \mu_{q}(h) d h d t \\
& =c \int_{0}^{\infty} \mu_{q}(h) \int_{0}^{1 / h} t^{1-(n-1) / q^{\prime}} d t d h \\
& =c^{\prime} \int_{0}^{\infty} h^{(n-1) / q^{\prime}-1}\left\|\Delta_{1}(h) f\right\|_{L^{q}\left[L^{1}\right]} \frac{d h}{h} \leq c^{\prime \prime}\|\nabla f\|_{1} .
\end{aligned}
$$

Further, set

$$
I^{\prime \prime}=\int_{0}^{\infty} s^{1 / n-1} \int_{s^{1 /(n-1)}}^{\infty} t^{1 / n-1} \Phi_{1}(s, t) d t d s .
$$

Now we choose $1<q<n^{\prime}$. Then, $1 / q^{\prime}<1 / n$. Applying (0.6), [4, Theorem 4.2], and (0.7), we get, as above

$$
\begin{aligned}
I^{\prime \prime} & \leq 2 \int_{0}^{\infty} s^{1 / n-1 / q^{\prime}-1} \int_{s^{1 /(n-1)}}^{\infty} t^{1 / n} \int_{0}^{1 / t} \mu_{q}(h) d h d t d s \\
& \leq 2 \int_{0}^{\infty} t^{1 / n} \int_{0}^{1 / t} \mu_{q}(h) d h \int_{0}^{t^{n-1}} s^{1 / n-1 / q^{\prime}-1} d s d t \\
& =c^{\prime} \int_{0}^{\infty} h^{(n-1) / q^{\prime}-1}\left\|\Delta_{1}(h) f\right\|_{L^{q}\left[L^{1}\right]} \frac{d h}{h} \leq c^{\prime \prime}\|\nabla f\|_{1} .
\end{aligned}
$$

Thus,

$$
\int_{0}^{\infty} \int_{0}^{\infty}(s t)^{1 / n-1} \Phi_{1}(s, t) d s d t=I^{\prime}+I^{\prime \prime} \leq c\|\nabla f\|_{1} .
$$

Similar estimates hold for any $j=1, \ldots, n$, and we obtain $(0.1)$.

Similarly, we have the following theorem.

Theorem 0.2 Let $f \in H W_{1}^{1}\left(\mathbb{R}^{2}\right)$. Then, 


$$
\int_{0}^{\infty} \int_{0}^{\infty}(s t)^{-1 / 2}\left[\Phi_{1}(s, t)+\Phi_{2}(s, t)\right] d s d t \leq c\left(\left\|D_{1} f\right\|_{H^{1}}+\left\|D_{2} f\right\|_{H^{1}}\right)
$$

where

$$
\Phi_{1}(s, t)=\sup _{\left|\xi_{1}\right| \geq t}\left(\widehat{(}_{\xi_{1}}\right)^{*}(s), \quad \Phi_{2}(s, t)=\sup _{\left|\xi_{2}\right| \geq t}\left(\widehat{f}_{\xi_{2}}\right)^{*}(s) .
$$

Let $f$ be an integrable function on $\mathbb{R}^{n}$. Observe that for any $j=1, \ldots, n$

$$
\Phi_{j}(s, t) \geq \mathcal{R}_{j} \widehat{f}(s, 2 t),
$$

where $\mathcal{R}_{j} \hat{f}(s, 2 t)$ is the iterated rearrangement of $\left|\widehat{f}\left(\xi_{j}, \widehat{\xi}_{j}\right)\right|$ obtained by rearranging first with respect to $\hat{\xi}_{j}$ and then with respect to $\xi_{j}$ (see [3]). Indeed, if $\varphi$ is a continuous rearrangeable function on $\mathbb{R}$, then

$$
\varphi^{*}(2 t) \leq \sup _{|x| \geq t}|\varphi(x)|, \quad t>0 .
$$

It is well known that for any function $g$ rearrangeable on $\mathbb{R}^{n}$ and any $j \in\{1, \ldots, n\}$

$$
\int_{0}^{\infty} t^{1 / n-1} g^{*}(t) d t \leq c \int_{0}^{\infty} \int_{0}^{\infty}(s t)^{1 / n-1} \mathcal{R}_{j} g(s, t) d s d t
$$

(see [3]). Thus, Theorem 0.1 gives a refinement of the inequality

$$
\int_{0}^{\infty} t^{1 / n-1} \widehat{f}^{*}(t) d t \leq c\|\nabla f\|_{1}
$$

proved in [1] (see also [2] for the estimates of iterated rearrangements).

For $k \in \mathbb{Z}$, denote

$$
P_{k}=\left\{y \in \mathbb{R}^{n-1}:\left|y_{m}\right| \leq 2^{k}, 1 \leq m \leq n-1\right\} .
$$

Corollary 0.3 Let $f \in W_{1}^{1}\left(\mathbb{R}^{n}\right)(n \geq 3)$. Then,

$$
\sum_{j=1}^{n} \sum_{k \in \mathbb{Z}} 2^{k(2-n)} \sup _{\left|\xi_{j}\right| \geq 2^{k}} \int_{P_{k+1}}|\widehat{f}(\xi)| d \widehat{\xi}_{j} \leq c|| \nabla f \|_{1} .
$$

Indeed, if $\left|\xi_{j}\right| \geq 2^{k}$ and $\mu_{k}=\operatorname{mes}_{n-1} P_{k}=2^{(k+1)(n-1)}$, then

$$
\int_{P_{k+1}}|\widehat{f}(\xi)| d \widehat{\xi}_{j} \leq \sup _{\left|\xi_{j}\right| \geq 2^{k}} \int_{0}^{\mu_{k+1}}\left(\widehat{f}_{\xi_{j}}\right)^{*}(s) d s \leq 2^{n-1} \int_{0}^{\mu_{k}} \Phi_{j}\left(s, 2^{k}\right) d s .
$$

Thus, estimate (0.8) is an immediate consequence of the inequality

$$
\sum_{j=1}^{n} \int_{0}^{\infty} t^{1-n} \int_{0}^{t^{n-1}} \Phi_{j}(s, t) d s d t \leq c\|\nabla f\|_{1}
$$

which in turn follows from Theorem 0.1 and the obvious inequality 


$$
\int_{0}^{\infty} \int_{0}^{\infty}(s t)^{1 / n-1} \Phi_{j}(s, t) d s d t \geq \int_{0}^{\infty} t^{1-n} \int_{0}^{t^{n-1}} \Phi_{j}(s, t) d s d t .
$$

Let $Q_{k}=\left[-2^{k}, 2^{k}\right]^{n}$. Since

$$
\begin{gathered}
\int_{\mathbb{R}^{n}}|\widehat{f}(\xi)||\xi|^{1-n} d \xi \leq c \sum_{k \in \mathbb{Z}} 2^{k(1-n)} \int_{Q_{k+1} \backslash Q_{k}}|\widehat{f}(\xi)| d \xi \\
\leq c^{\prime} \sum_{j=1}^{n} \sum_{k \in \mathbb{Z}} 2^{k(2-n)} \sup _{2^{k} \leq\left|\xi_{j}\right| \leq 2^{k+1}} \int_{P_{k+1}}|\widehat{f}(\xi)| d \hat{\xi}_{j},
\end{gathered}
$$

(0.8) gives the strengthening of the inequality [4, (6.2)] (for $n \geq 3$ ).

Estimate $(0.8)$ can be interpreted as an Oberlin-type estimate for the surfaces of $n$-dimensional cubes. There holds also the following

Theorem 0.4 Let $f \in W_{1}^{1}\left(\mathbb{R}^{n}\right)(n \geq 2)$. Then,

$$
\sum_{k \in \mathbb{Z}} 2^{k(2-n)} \sup _{2^{k} \leq r \leq 2^{k+1}} \int_{S_{r}}|\widehat{f}(\xi)| d \sigma(\xi) \leq c\|\nabla f\|_{1},
$$

where $S_{r}$ is the sphere of the radius $r$ centered at the origin in $\mathbb{R}^{n}$ and $d \sigma(\xi)$ is the canonical surface measure on $S_{r}$.

This theorem was formulated for $n \geq 3$ in our work [4, Theorem 6.5].

However, the proof given there is invalid. Indeed, this proof was based on the inequality [4, (6.5)] with $F_{t, j}$ defined by [4, (6.6)]. It was a mistake because really [4, (6.5)] was proved for $F_{t, j}$ defined in a different way (namely, taking first rearrangement with respect to $\hat{\xi}_{j}$, and then the supremum with respect to $\xi_{j}$ ).

The complete proof of Theorem 0.4 was given by Stolyarov [5] with the use of the Tomas-Stein restriction theorem [6]. The study of Besov spaces in [5] was based on the Peetre definition. For the completeness, we give a sketch of the proof from [5] with a modification corresponding to the classical definition of Besov spaces (in terms of moduli of continuity).

Fix $r>0$. Let $S_{r, j}=\left\{\xi \in S_{r}:\left|\xi_{j}\right| \geq r / \sqrt{n}\right\}$. Then, $S_{r}=\bigcup_{j=1}^{n} S_{r, j}$. Take $j=1$ and set $\varphi_{h}(x)=\Delta_{1}(h) f(x), v=\sqrt{n} / r$. As in Theorem 0.1,

$$
\frac{2}{v} \int_{0}^{v}\left|\widehat{\varphi_{h}}(\xi)\right| d h \geq|\widehat{f}(\xi)| \quad \text { if } \quad\left|\xi_{1}\right| \geq r / \sqrt{n} .
$$

Thus,

$$
\begin{aligned}
\int_{S_{r, j}}|\widehat{f}(\xi)| d \sigma(\xi) \leq \frac{2}{v} \int_{0}^{v} \int_{S_{r}}\left|\widehat{\varphi}_{h}(\xi)\right| d \sigma(\xi) d h \\
=\frac{2}{\sqrt{n}} \int_{0}^{v} \int_{S_{1}}\left|\widehat{\psi}_{h, r}(\xi)\right| d \sigma(\xi) d h, \quad \text { where } \quad \psi_{h, r}(x)=\varphi_{h}(x / r) .
\end{aligned}
$$

Let $1<q<2(n+1) /(n+3)$. By the Tomas-Stein theorem [6], 


$$
\int_{S_{1}}\left|\widehat{\psi}_{h, r}(\xi)\right| d \sigma(\xi) \leq c\left(\int_{\mathbb{R}^{n}}\left|\psi_{h, r}(x)\right|^{q} d x\right)^{1 / q}=c r^{n / q}|| \varphi_{h} \|_{q} .
$$

It follows that

$$
\max _{r / 2 \leq \rho \leq r} \int_{S_{\rho}}|\widehat{f}(\xi)| d \sigma(\xi) \leq c r^{n / q} \sum_{j=1}^{n} \int_{0}^{2 v}\left\|\Delta_{j}(h) f\right\|_{q} d h .
$$

Applying this estimate and [4, Theorem 1.2] (we may assume that $q<n^{\prime}$ ), we easily obtain (0.9).

We observe that similar reasonings cannot be applied in Corollary 0.3. Indeed, in particular, it is easily seen that restriction theorems are not true for line segments in $\mathbb{R}^{2}$. The question on the validity of inequality (0.8) for $n=2$ remains open.

Acknowledgements The author is grateful to the referee for his/her useful remarks.

\section{References}

1. Kolyada, V.I.: Estimates of Fourier transforms in Sobolev spaces. Studia Math. 125, 67-74 (1997)

2. Kolyada, V.I.: Embeddings of fractional Sobolev spaces and estimates of Fourier transforms. Mat. Sb. 192(7), 51-72 (2001) [English transl.: Sbornik: Mathematics 192(7), 979 - 1000 (2001)]

3. Kolyada, V.I.: On embedding theorems, In: Nonlinear Analysis, Function Spaces and Applications, vol. 8 (Proceedings of the Spring School held in Prague, 2006), Prague, pp. 35-94 (2007)

4. Kolyada, V.I.: Embedding theorems for Sobolev and Hardy-Sobolev spaces and estimates of Fourier transforms. Ann. Mat. Pura Appl. 198(2), 615-637 (2019)

5. Stolyarov, D.: Hardy-Littlewodd-Sobolev inequality for $p=1$. arXiv:2010.05297v3 [math.CA], 5 Feb 2021

6. Tomas, P.A.: A restriction theorem for the Fourier transform. Bull. Am. Math. Soc. 81(2), 477-478 (1975)

Publisher's Note Springer Nature remains neutral with regard to jurisdictional claims in published maps and institutional affiliations. 